\section{Comments on "Least Squares Restoration of Multichannel Images"}

Michael K. Ng and Wilson C. Kwan

Abstract-In this correspondence, we give the correct matrix formulation arising from the constrained optimization of the least squares restoration of multichannel images in the above paper. ${ }^{1}$

Index Terms-Image restoration, least squares method.

The degradation of multichannel images can be modeled as solving

$$
\mathbf{g}=\mathbf{H} \mathbf{f}+\mathbf{n}
$$

where

H degradation matrix;

n unknown Gaussian noise or measurement errors;

g observed multichannel image;

f original multichannel image.

For $N$ channels of $M \times M$ pixels each, the observed and original images can be expressed as

$$
\mathbf{g}=\left(\begin{array}{c}
\mathbf{g}_{1} \\
\mathbf{g}_{2} \\
\vdots \\
\mathbf{g}_{N}
\end{array}\right), \quad \mathbf{f}=\left(\begin{array}{c}
\mathbf{f}_{1} \\
\mathbf{f}_{2} \\
\vdots \\
\mathbf{f}_{N}
\end{array}\right)
$$

where each of the $M^{2}$-vectors $\mathbf{g}_{i}$ and $\mathbf{f}_{i}$ are the observed and the original images in each channel. The multichannel degradation operator $\mathbf{H}$ is given by

$$
\mathbf{H}=\left(\begin{array}{cccc}
\mathbf{H}_{11} & \mathbf{H}_{12} & \cdots & \mathbf{H}_{1 N} \\
\mathbf{H}_{21} & \mathbf{H}_{22} & \cdots & \mathbf{H}_{2 N} \\
\vdots & \cdots & \ddots & \vdots \\
\mathbf{H}_{N 1} & \mathbf{H}_{N 2} & \cdots & \mathbf{H}_{N N}
\end{array}\right) .
$$

Here, the operators $\mathbf{H}_{i i}$ and $\mathbf{H}_{i j}(i \neq j)$ represent the within-channel and the cross-channel degradation operators, respectively.

As the system (1) is ill-conditioned and generally not positive definite, we solve it by using a constrained minimization technique. The solution $\hat{\mathbf{f}}$ is determined by minimizing $\|\mathbf{Q} \mathbf{f}\|_{2}^{2}$ subject to $\left\|\overline{\mathbf{H}}_{i}-\mathbf{g}_{i}\right\|_{2}^{2}=$ $\left\|\mathbf{n}_{i}\right\|_{2}^{2}$ for $1 \leq i \leq N$, where

$$
\overline{\mathbf{H}}_{i}=\left(\mathbf{H}_{i 1}, \mathbf{H}_{i 2}, \ldots, \mathbf{H}_{i N}\right) \text {. }
$$

Using the method of Lagrange multipliers, the solution is obtained by minimizing

$$
\Phi(\mathbf{f}, \lambda)=\sum_{i=1}^{N} \lambda_{i}\left(\left\|\overline{\mathbf{H}}_{i} \mathbf{f}-\mathbf{g}_{i}\right\|_{2}^{2}-\left\|\mathbf{n}_{i}\right\|_{2}^{2}\right)+\|\mathbf{Q f}\|_{2}^{2}
$$

where $\lambda=\left(\lambda_{1}, \lambda_{2}, \ldots, \lambda_{N}\right)^{t}$ is the Lagrange multiplier vector. The necessary conditions for the solution of (2) are

$$
\nabla_{f} \Phi(\mathbf{f}, \lambda)=\mathbf{0}
$$

Manuscript received January 18, 2000; revised July 27, 2001. This work was supported in part by the Research Grant Council under HKU 7147/99P and 7132/00P and HKU CRCG Grant 10202720 and 10203501. The associate editor coordinating the review of this paper and approving it for publication was Prof. Colin F. Cowan.

The authors are with the Department of Mathematics, The University of Hong Kong, Hong Kong (e-mail: mng@maths.hku.hk).

Publisher Item Identifier S 1053-587X(01)09222-4.

${ }^{1}$ N. Galatsanos, A. Katsaggelos, R. Chin, and A. Hillery, "Least squares restoration of multichannel images," IEEE Trans. Signal Processing, vol. 39, pp. 2222-2236, Oct. 1991.

$$
\nabla_{\alpha} \Phi(\mathbf{f}, \lambda)=\mathbf{0}
$$

From (3), we have

$$
\left[\sum_{i=1}^{N} \lambda_{i} \overline{\mathbf{H}}_{i}^{t} \overline{\mathbf{H}}_{i}+\mathbf{Q}^{\mathbf{t}} \mathbf{Q}\right] \hat{\mathbf{f}}=\sum_{i=1}^{N} \lambda_{i} \overline{\mathbf{H}}_{i}^{t} \mathbf{g}_{i} .
$$

Since

$$
\sum_{i=1}^{N} \lambda_{i} \overline{\mathbf{H}}_{i}^{t} \overline{\mathbf{H}}_{i}=\mathbf{H}^{t} \boldsymbol{\Lambda} \mathbf{H}
$$

where $\boldsymbol{\Lambda}$ is defined as

$$
\boldsymbol{\Lambda}=\left(\begin{array}{cccc}
\lambda_{1} \mathbf{I} & 0 & \cdots & 0 \\
0 & \lambda_{2} \mathbf{I} & \cdots & 0 \\
\vdots & \cdots & \ddots & \vdots \\
0 & 0 & \cdots & \lambda_{N} \mathbf{I}
\end{array}\right)
$$

the matrix system should be

$$
\left[\mathbf{H}^{\mathbf{t}} \boldsymbol{\Lambda} \mathbf{H}+\mathbf{Q}^{\mathbf{t}} \mathbf{Q}\right] \hat{\mathbf{f}}=\Lambda \mathbf{H}^{\mathbf{t}} \mathbf{g}
$$

instead of

$$
\left[\Lambda \mathbf{H}^{\mathbf{t}} \mathbf{H}+\mathbf{Q}^{\mathbf{t}} \mathbf{Q}\right] \hat{\mathbf{f}}=\boldsymbol{\Lambda} \mathbf{H}^{\mathbf{t}} \mathbf{g}
$$

which is stated in (2.14) of the paper. We remark that if there are only the within-channel blur and no cross-channel blur in the degradation, i.e., $H_{i j}=0$, then both formulae (5) and (6) are identical.

Solving for $\hat{\mathbf{f}}$ in (5) yields

$$
\hat{\mathbf{f}}=\left[\mathbf{H}^{\mathbf{t}} \boldsymbol{\Lambda} \mathbf{H}+\mathbf{Q}^{\mathbf{t}} \mathbf{Q}\right]^{-\mathbf{1}} \boldsymbol{\Lambda} \mathbf{H}^{\mathbf{t}} \mathbf{g}
$$

which is the solution of (2) with respect to chosen values in $\lambda$. We note in the paper that the incorrect solution is given by

$$
\hat{\mathbf{f}}=\left[\mathbf{H}^{\mathrm{t}} \mathbf{H}+\boldsymbol{\Lambda}^{-1} \mathbf{Q}^{\mathrm{t}} \mathbf{Q}\right]^{-1} \mathbf{H}^{\mathbf{t}} \mathbf{g},
$$

[see (2.15) of the paper].

As the solution $\hat{\mathbf{f}}$ must satisfy the following functions:

$$
\begin{array}{r}
Z_{i}\left(\lambda_{1}, \lambda_{2}, \ldots, \lambda_{N}\right)=\left\|\overline{\mathbf{H}}_{i} \hat{\mathbf{f}}-\mathbf{g}_{i}\right\|_{2}^{2}-\left\|\mathbf{n}_{i}\right\|_{2}^{2}, \\
\quad i=1,2, \ldots, N
\end{array}
$$

finding the roots of the functions $Z_{i}$ simultaneously yields the desired $\lambda_{i}$. Since the functions $Z_{i}$ are nonlinear, Newton's method is therefore used to find these $\lambda_{i}$ numerically. Since Newton's method for the estimate of $\lambda_{i}$ is derived according to the form of $\hat{f}$, the correct formula of $\hat{f}$ is important in the estimation of $\lambda_{i}$ and the restoration of multichannel images.

The $(i, j)$ th element of the Jacobian matrix $\mathbf{J}$ of this nonlinear system of functions based on the matrix formulation (5) should be given by

$$
[\mathbf{J}]_{i j}=\frac{\partial Z_{i}\left(\lambda_{1}, \lambda_{2}, \ldots, \lambda_{N}\right)}{\partial \lambda_{j}}=2\left(\overline{\mathbf{H}}_{i} \hat{\mathbf{f}}-\mathbf{g}_{i}\right)^{t} \overline{\mathbf{H}}_{i} \frac{\partial \hat{\mathbf{f}}}{\partial \lambda_{j}}
$$

for $1 \leq i, j \leq N$. By (7), we obtain

$$
\begin{aligned}
\frac{\partial \hat{\mathbf{f}}}{\partial \lambda_{j}}=\left[\mathbf{H}^{\mathbf{t}} \boldsymbol{\Lambda} \mathbf{H}\right. & \left.+\mathbf{Q}^{\mathbf{t}} \mathbf{Q}\right]^{-\mathbf{1}} \\
& \cdot\left(\mathbf{I}_{j j}-\overline{\mathbf{H}}_{j}^{t} \overline{\mathbf{H}}_{j}\left[\mathbf{H}^{\mathbf{t}} \boldsymbol{\Lambda} \mathbf{H}+\mathbf{Q}^{\mathbf{t}} \mathbf{Q}\right]^{-\mathbf{1}} \boldsymbol{\Lambda}\right) \mathbf{H}^{\mathbf{t}} \mathbf{g}
\end{aligned}
$$

where $\mathbf{I}_{j j}$ is a zero matrix, except that the $(j, j)$ th diagonal block is an identity matrix. Hence, we have

$$
\begin{aligned}
{[\mathbf{J}]_{i j}=2\left(\overline{\mathbf{H}}_{i} \hat{\mathbf{f}}-\mathbf{g}_{i}\right)^{t} \overline{\mathbf{H}}_{i}\left[\mathbf{H}^{\mathbf{t}} \boldsymbol{\Lambda} \mathbf{H}+\mathbf{Q}^{\mathbf{t}} \mathbf{Q}\right]^{-\mathbf{1}} } & \cdot\left(\mathbf{I}_{j j}-\overline{\mathbf{H}}_{j}^{t} \overline{\mathbf{H}}_{j}\left[\mathbf{H}^{\mathbf{t}} \boldsymbol{\Lambda} \mathbf{H}+\mathbf{Q}^{\mathbf{t}} \mathbf{Q}\right]^{-\mathbf{1}} \boldsymbol{\Lambda}\right) \mathbf{H}^{\mathbf{t}} \mathbf{g}
\end{aligned}
$$

which is the correct formula for the Jacobian matrix. 